\title{
Abelhas (Hymenoptera: Apoidea) visitantes dos capítulos de girassol no recôncavo baiano
}

\author{
Bees (Hymenoptera: Apoidea) on sunflower flowers in Recôncavo Baiano region, Brazil
}

\author{
Cerilene Santiago Machado ${ }^{1}$ Carlos Alfredo Lopes de Carvalho
}

\section{RESUMO}

O girassol (Helianthus annuus L.) é uma espécie de polinização cruzada, cujos principais polinizadores são as abelhas. Este trabalho teve por objetivo obter informações sobre a diversidade de abelhas visitantes nos capítulos de girassol, fornecendo subsídios para identificar os possíveis polinizadores efetivos na região do Recôncavo Baiano. As coletas das abelhas foram realizadas no período de setembrooutubro de 2004, janeiro-fevereiro e maio-junho de 2005 sobre os híbridos de girassol, "AG 920” e "AG 930”, cultivados na área experimental de Entomologia do Centro de Ciências Agrárias e Ambientais da Universidade Federal da Bahia, em Cruz das Almas-BA. Um total de 26 espécies de abelhas foram identificadas, sendo que Apis mellifera foi a mais abundante (58,32\%), seguida de Nannotrigona testaceicornis (18,53\%) e Trigona spinipes $(11,37 \%)$. As demais espécies apresentaram uma freqüência relativa inferior a $5 \%$.

Palavras-chave: Helianthus annuus; Apoidea; Apis mellifera; Nannotrigona testaceicornis.

\section{ABSTRACT}

Sunflower, Helianthus annuus L., is a crosspollinating plant whose main pollinator are bees. This work was airmed at getting information about the diversity of visiting bee on sunflower inflorescence, by identifing the possible sunflower pollinators in Recôncavo Baiano region. The bees were collected on September-October of 2004, JanuaryFebruary and May-June of 2005 sunflowers hybrids (AG 920 and AG 930) cultivated in the entomology experimental area of Centro de Ciências Agrárias e Ambiental da Universidade Federal da Bahia, Brazil. Twenty six species were identified Apis mellifera (58.32\%), Nannotrigona testaceicornis (18.53\%) and Trigona spinipes (11.37\%) were the most abundant species. The other species presented a frequency lower than relative $5 \%$.
Key words: Helianthus annuus; Apoidea; Apis mellifera; Nannotrigona testaceicornis.

\section{INTRODUÇÃO}

O girassol (Helianthus annuus L.) é uma planta dicotiledônea anual, cujo arranjo floral permite que seja beneficiado quando é visitado por abelhas coletoras de néctar. Isso ocorre porque a sua inflorescência constitui-se em um capítulo cujas flores abrem em seqüência de fora para dentro, ao longo de vários dias (McGREG0R, 1976).

As flores passam primeiro por uma fase masculina, na qual o pólen é liberado, e logo em seguida por uma fase feminina, quando se tornam receptivos os estigmas. Dessa forma, as abelhas que coletam pólen limitam suas visitas às flores em fase masculina, enquanto que as abelhas coletoras de néctar visitam todas as flores da inflorescência, efetuando a polinização cruzada (FREE, 1993).

As flores de girassol são uma boa fonte de néctar às abelhas (SCHINOHARA et al., 1987). O néctar produzido pelas flores do girassol pode ser um aspecto importante na estratégia de atração e manutenção de polinizadores em áreas cultivadas, assim como pode contribuir para o aumento da produção de mel explorado por apicultores e meliponicultores.

Estudos sobre a influência de visitas de abelhas nas inflorescências dessa planta indicam que estas são extremamente importantes na polinização,

${ }^{1}$ Departamento de Fitotecnia, Centro de Ciências Agrárias, Universidade Federal da Bahia (UFBA), CP 118, 44380-000, Cruz das Almas, BA, Brasil.

${ }^{2}$ Departamento de Fitotecnia, Centro de Ciências Agrárias, UFBA, CP 118, 44380-000, Cruz das Almas, BA, Brasil. E-mail: calfredo@ufba.br. Autor para correspondência. 
aumentando a produção e a qualidade das sementes (MORETI, 1989; SILVA, 1990).

O cultivo de girassol em diferentes regiões agrícolas do Estado da Bahia tem despertado o interesse de empresários devido a sua versatilidade de utilização e, principalmente, devido à possibilidade de sua utilização como óleo combustível, uma vez que este Estado é considerado pólo estratégico para o desenvolvimento do Probiodiesel (DINHEIRO RURAL, 2005; REDE BAIANA DE BIOCOMBUSTÍVEL, 2006).

Considerando a importância econômica da cultura e a falta de informações sobre os polinizadores do girassol no Recôncavo Baiano, este trabalho foi desenvolvido com o objetivo de determinar a diversidade e a abundância de abelhas que visitam o girassol, para identificar os possíveis polinizadores potenciais na região.

\section{MATERIAL E MÉTODOS}

O trabalho foi conduzido na área experimental de entomologia do Centro de Ciências Agrárias da Universidade Federal da Bahia, localizada no município de Cruz das Almas, Bahia, situado no Recôncavo Baiano, a 12 40' 39" latitude sul, 39 40' 23" longitude oeste de Greenwich, com altitude de $220 \mathrm{~m}$ acima do nível do mar, temperatura média anual de $24,5^{\circ} \mathrm{C}$, umidade relativa de $80 \%$ e precipitação pluvial média de $1.224 \mathrm{~mm}$. Segundo a classificação de Köpen, o clima é tropical quente úmido, AW a AM (ALMEIDA, 1999).

Durante o período compreendido entre junho de 2004 a julho de 2005, foram realizados três plantios de girassol em uma área de $1000 \mathrm{~m}^{2}$, utilizando espaçamento com $0,5 \mathrm{~m}$ entre plantas e $0,9 \mathrm{~m}$ entre linhas, conforme indicado por INÁCIO et al. (2003).

Os híbridos utilizados nos plantios foram o “AG 920” e o “AG 930” da empresa AGROBEL, que tem alturas de plantas de aproximadamente 1,80 a 1,90m e ciclos de 95 a 125 dias.

Os espécimes foram coletados quando visitavam as inflorescências dos híbridos de girassol dos três plantios, durante os meses de setembrooutubro de 2004, janeiro-fevereiro e maio-junho de 2005, totalizando 58 coletas. Em cada plantio foram definidos aleatoriamente 20 capítulos por híbrido e por intervalo de hora para a coleta efetiva das abelhas. As coletas foram realizadas entre as $6 \mathrm{~h}$ e as $18 \mathrm{~h}$.

Os insetos foram coletados com sacos plásticos transparentes, separados em morfoespécies e catalogados. A identificação dos espécimes foi baseada nos exemplares depositados no Museu Entomológico da Escola de Agronomia da UFBA.

A análise da fauna de abelhas foi baseada na freqüência relativa de indivíduos das diferentes espécies (SILVEIRA-NETO, 1976), na dominância (KATO et al., 1952) e nos seguintes índices faunísticos: abundância de Margalef (a), índices de diversidade de Shannon-Wiener (H') e de Simpson (l) e índice de eqüitabilidade de Pielou (J'), conforme descrições em LUDWIG \& REYNOLDS (1988). A similaridade entre a composição de espécies visitantes nos dois híbridos foi determinada pelo Coeficiente de Sprense (BROWER \& ZAR, 1984).

\section{RESULTADOS E DISCUSSÃO}

Nas coletas, foram capturadas 25.562 abelhas de 26 espécies, que correspondem a 18 gêneros, seis tribos e três famílias (Tabela 1). A família Apidae sensu ROIG-ALSINA \& MICHENER (1994) representou 99,95\% dos indivíduos e 80,77\% das espécies. Os gêneros Melipona, Trigona e Xylocopa, com três espécies, foram os de maior riqueza de espécies.

A similaridade de riqueza de espécies de abelhas entre os dois híbridos de girassol foi elevada, pois o Coeficiente de Sфrense, para um valor máximo teórico de 1,0, foi de 0,84 .

Apis mellifera foi a espécie mais abundante, com freqüência relativa de 58,32\% dos espécimes, seguida de Nannotrigona testaceicornis (18,53\%) e Trigona spinipes (11,37\%). Essas três espécies foram consideradas dominantes. As demais espécies apresentaram uma freqüência relativa inferior a 5\%.

Apis mellifera tem sido relatada como principal polinizador do girassol em diferentes estudos (McGREGOR, 1976; PARKER, 1981; MORETI et al., 1991; MORETI \& MARCHINI, 1992; DAG et al., 2002; MORETI, 2005), alcançando freqüência relativa superior a 93\% (BUTIGNOL, 1990).

Nannotrigona testaceicornis é uma espécie abundante em diferentes culturas da região 
Tabela 1 - Abelhas (Apoidea)* visitantes de capítulos de girassol (Helianthus annuus) no Recôncavo Baiano: setembro-outubro de 2004, janeiro-fevereiro e maio-junho de 2005.

\begin{tabular}{|c|c|c|c|}
\hline Família / Subfamília / Tribo / Subtribo / Espécie & № de indivíduos & Freqüência Relativa (\%) & Constância nas coletas (\%) \\
\hline \multicolumn{4}{|l|}{ Apidae } \\
\hline \multicolumn{4}{|l|}{ Apinae } \\
\hline \multicolumn{4}{|l|}{ Apini } \\
\hline \multicolumn{4}{|l|}{ Apina } \\
\hline Apis mellifera L. 1758 & 14.907 & 58,317 & 98,36 \\
\hline \multicolumn{4}{|l|}{ Bombina } \\
\hline Bombus atratus Franklin, 1913 & 5 & 0,020 & 8,20 \\
\hline \multicolumn{4}{|l|}{ Euglossina } \\
\hline Euglossa cordata (Linnaeus, 1758) & 1 & 0,004 & 1,64 \\
\hline Eulaema nigrita Lepeletier,1841 & 3 & 0,012 & 4,92 \\
\hline \multicolumn{4}{|l|}{ Meliponina } \\
\hline Friesomellita sp. & 1 & 0,004 & 1,64 \\
\hline Melipona asilvai Moure, 1971 & 1 & 0,004 & 1,64 \\
\hline M. quadrifasciata Lepeletier,1936 & 548 & 2,144 & 75,41 \\
\hline M. scutellaris Latreille,1811 & 620 & 2,425 & 80,33 \\
\hline Nannotrigona testaceicornis (Lepeletier,1936) & 4.736 & 18,528 & 96,72 \\
\hline Partamona helleri (Friese,1900) & 119 & 0,466 & 47,54 \\
\hline Scaptotrigona sp. & 18 & 0,070 & 14,75 \\
\hline Tetragonisca angustula (Latreille,1811) & 706 & 2,762 & 83,61 \\
\hline Trigona fuscipennis Friese, 1900 & 75 & 0,293 & 34,43 \\
\hline T. hyalinata (Lepeletier,1836) & 798 & 3,122 & 86,89 \\
\hline T. spinipes (Fabricius,1793) & 2.906 & 11,368 & 90,16 \\
\hline \multicolumn{4}{|l|}{ Eucerini } \\
\hline Melissodes nigroaeneae (Smith,1852) & 3 & 0,012 & 3,28 \\
\hline \multicolumn{4}{|l|}{ Exomalopsini } \\
\hline Exomalopsis analis Spinola, 1853 & 63 & 0,246 & 47,54 \\
\hline E. auropilosa Spinola, 1853 & 32 & 0,125 & 31,15 \\
\hline \multicolumn{4}{|l|}{ Xylocopinae } \\
\hline \multicolumn{4}{|l|}{ Xylocopini } \\
\hline Xylocopa carbonaria Smith, 1854 & 2 & 0,008 & 3,28 \\
\hline X. suspecta Moura \& Camargo, 1988 & 4 & 0,016 & 6,56 \\
\hline X. frontalis (Olivier,1789) & 1 & 0,004 & 1,64 \\
\hline \multicolumn{4}{|l|}{ Halictidae } \\
\hline \multicolumn{4}{|l|}{ Halictinae } \\
\hline \multicolumn{4}{|l|}{ Augochlorini } \\
\hline Augochlorella ephyra (Schrottky, 1910) & 2 & 0,008 & 1,64 \\
\hline Augochloropsis callichroa (Cockerell) & 3 & 0,012 & 4,92 \\
\hline Pereirapis sp. & 1 & & 1,64 \\
\hline \multicolumn{4}{|l|}{ Megachilidae } \\
\hline \multicolumn{4}{|l|}{ Megachilinae } \\
\hline \multicolumn{4}{|l|}{ Megachilini } \\
\hline Megachile paulistana Schrottky,1902 & 6 & 0,023 & 9,84 \\
\hline Megachile sp. & 1 & 0,004 & 1,64 \\
\hline
\end{tabular}

*Classificação conforme Silveira et al., 2002.

(CARVALHO et al., 2000; CARVALHO et al., 2002; BARROS et al., 2002).

Trigona spinipes é relatada como espécie abundante em outros estudos (AGUIAR \& MARTINS, 1997; SANTOS et al., 2004). Segundo ALMEIDA \&
LAROCA (1988), essa espécie apresenta algumas características que favorecem sua abundância em vários habitats, como a agressividade de suas campeiras, ninhos construídos em diferentes locais de difícil acesso, hábito generalista de coleta e colônias 
populosas. Essa espécie é considerada praga em diversas culturas por causar danos durante a coleta de tecido vegetal utilizado na construção dos seus ninhos (GALLO et al., 2002). Conforme MORGADO et al. (2002), em plantios de girassol de Lavras-MG, T. spinipes foi mais abundante que $\boldsymbol{A}$. mellifera em determinadas épocas do ano.

A freqüência elevada de indivíduos de $N$. testacecornis e $\mathbf{T}$. spinipes pode ser explicada pela população elevada dos ninhos dessas espécies e pela presença de colônias próximo da área plantada com girassol. Por outro lado, é possível que ocorra afinidade entre essas espécies e o girassol, uma vez que colônias de outras espécies generalistas de meliponíneos encontravam-se próximas da mesma área e não apresentaram valores elevados de freqüência relativa nos capítulos de girassol.

As outras espécies coletadas são comuns na área e a maioria já foi registrada como visitantes das inflorescências do girassol (BUTIGNOL, 1990; MORETI \& MARCHINI, 1992; MORGADO et al., 2002; PAIVA et al., 2002). Apenas duas espécies de Megachile foram coletadas. Espécies desse gênero também foram registradas em girassol por MORETI \& MARCHINI (1992) e MORGADO et al. (2002). Megachile squalens foi a segunda espécie mais abundante no girassol em Florianópolis-SC (BUTIGNOL, 1990). De acordo com IGLESIAS (1984), espécies de Megachile possuem potencial para serem manejadas visando à polinização do girassol.

O reduzido número de espécies solitárias pode estar relacionado com o uso intensivo da área cultivada, o que reduz as opções de locais para nidificação.

Levantamentos de abelhas realizados em diferentes culturas na mesma área indicam uma baixa freqüência de abelhas solitárias e destacam a abundância de meliponíneos, entre elas $N$. testaceicornis e T. angustula (CARVALHO et al., 2000; CARVALHO et al., 2002; BARROS et al., 2002).

Considerando a constância das espécies ao longo do período de florescimento do girassol, pelo menos sete espécies foram coletadas em mais de 70\% das amostragens. Os índices faunísticos (Tabela 2) permitem fazer as seguintes observações sobre a comunidade de abelhas: o índice $\alpha$ obtido indica que ocorreu competição interespecífica, enquanto que os índices de diversidade ( $H$ ’ e $\lambda$ ) permitem inferir sobre uma provável perda de diversidade de abelhas e o índice J' confirma o desequilíbrio entre o número de indivíduos por espécie (conforme LUDWIG \& REYNOLDS, 1988; ODUM, 1988).

Comparando a diversidade de gêneros de abelhas visitantes do girassol em quatro regiões brasileiras (Tabela 3), observa-se uma maior semelhança entre a fauna de abelhas nos plantios de girassol em Lavras-MG e em Piracicaba-SP. Apenas os gêneros Apis, Megachile, Trigona e Xylocopa foram representados nos quatros estudos. Representantes de Trigona e de Apis também foram coletados por PAIVA et al. (2002) em Maringá-PR.

De acordo com GRANDI-HOFFMAN \& WATKINS (2000), a combinação entre A. mellifera e outras espécies de abelhas resulta em uma melhora da polinização em híbridos de girassol.

\section{CONCLUSÃO}

As principais espécies visitantes de girassol no Recôncavo Baiano foram Apis mellifera, Nannotrigona testaceicornis e Trigona spinipes, sendo consideradas como potenciais polinizadores dessa cultura na região.

\section{AGRADECIMENTOS}

Ao Conselho Nacional de Desenvolvimento Científico e Tecnológico (CNPq), pela concessão de bolsa de Mestrado (MSc) ao autor Machado e pela bolsa de Produtividade em Pesquisa ao autor Carvalho.

Tabela 2 - Índices de abundância $(\alpha)$, de diversidade (H’ e $\lambda$ ) e de eqüitabilidade da comunidade de abelhas (Hymenoptera: Apoidea) visitantes dos capítulos de girassol no Recôncavo Baiano: setembro-outubro de 2004, janeiro-fevereiro e maio-junho de 2005.

\begin{tabular}{lc}
\hline Índices & Valores obtidos \\
\hline$\alpha$ (Margalef) & 2,46 \\
H' (Shannon-Wiener) & 1,34 \\
$\lambda$ (Simpson) & 0,39 \\
J' (Pielou) & 0,41 \\
\hline
\end{tabular}


Tabela 3 - Similaridade entre a diversidade de gêneros de abelhas visitantes dos capítulos de girassol em quatro localidades do Brasil por meio do Coeficiente de S申rense.

\begin{tabular}{lcccc}
\hline Localidades & Presente estudo & $\begin{array}{c}\text { Florianópolis - SC } \\
\text { (Butignol, 1990) }\end{array}$ & $\begin{array}{c}\text { Piracicaba - SP } \\
\text { (Moreti \& Marchini, 1992) }\end{array}$ & $\begin{array}{c}\text { Lavras - MG } \\
\text { (Morgado et al., 2002) }\end{array}$ \\
\hline Presente estudo & - & 0,32 & 0,51 & 0,47 \\
Florianópolis-SC & - & 0,42 & 0,38 \\
Piracicaba-SP & & - & 0,57 \\
\hline
\end{tabular}

\section{REFERÊNCIAS}

AGUIAR, C.M.L.; MARTINS, C.F. Abundância relativa, diversidade e fenologia de abelhas (Hymenoptera, Apoidea) na caatinga, São João do Cariri, Paraíba, Brasil. Iheringia, Porto Alegre, n.83, p.151-163, 1997. (Série Zoologia).

ALMEIDA, M.C. de; LAROCA, S. Trigona spinipes (Apidae, Meliponinae): taxonomia, bionomia e relação tróficas em áreas restritas. Acta Biológica Paranaense, Curitiba, v.17, n.1/4, p.67-108, 1988.

ALMEIDA, O.A. Informações metereológicas do CNP: mandioca e fruticultura tropical. Cruz das Almas-Ba: EMBRAPA-CNPMF, 1999. 35p. (Documentos, 34).

BARROS, T.F. et al. Abelhas visitantes de flores de Pimpinela anisum L. Magistra, Cruz das Almas, v.14, n.1, p.55-60, 2002.

BROWER, J.E.; ZAR, J.H. Field and laboratory methods for general ecology. 2.ed. Dubuque: Wm. C. Brown, 1984. 255p.

BUTIGNOL, C.A. Ocorrência de insetos em capítulos de girassol em distintos horários e estágios de florescimento. Anais da Sociedade de Entomológica do Brasil, Porto Alegre, v.19, n.2, p.273-280, 1990.

CARVALHO, C.A.L. de et al. Entomofauna visitante das flores do Jambeiro (Eugenia malaccensis L.) em Cruz das Almas-BA. In: CONGRESSO BRASILEIRO DE APICULTURA, 14., 2002, Campo Grande-MS. Anais... Campo Grande-MS: CBA, 2002. CD-ROM.

CARVALHO, C.A.L. de et al. Atividade forrageira de Nannotrigona punctata Smith nas flores de Cariondrum sativum (Apiaceae). In: ENCONTRO SOBRE ABELHAS, 4., 2000, Ribeirão Preto-SP. Anais... Ribeirão Preto-SP: FFCLRP-USP, 2000. p.341.

DAG, A. et al. Pollination of confection sunflowers (Helianthus annuus L.) by honey bees (Apis mellifera L.). American Bee Journal, Hamilton, v.142, n.6, p.443-445, 2002.

DINHEIRO RURAL. Bahia de todos os campos. Dinheiro Rural, São Paulo, v.2, n.9, p.67-89, 2005.

FREE, J.B. Insect pollination of crops. London: Academic, 1993. 684p.

GALLO, D. et al. Entomologia agrícola. São Paulo: FEALQ, 2002. 920p.
GRANDI-HOFFMAN, G. de; WATKINS, J.C. The foraging activity of honey bees Apis mellifera and non-Apis bees on hybrid sunflowers (Helianthus annuus) and its influence on cross-pollination and seed set. Journal of Apicultural Research, Georgia, v.39, n.1/2, p.37-45, 2000.

IGLESIAS, S.W. Himenopteros polinizadores y su efecto em contenido de aceite y rendimiento em girasol (Helianthus annuus L.). Oleico, v.26, p.11-13, 1984.

INÁCIO, F.R. et al. Influência de diferentes espaçamentos de plantio na visitação de Apis mellifera L. e na produtividade da cultura do girassol (Helianthus annuus L.). Magistra, Cruz das Almas, v.15, n.1, p. 93-96, 2003.

KATO, M. et al. Associative ecology of insects found in paddy field cultivated by various planning forms. Scientific Report of Tohoku University, Sendai, v.9, p.291-301, 1952. (Serie Biology,4).

LUDWIG, J.A.; REYNOLDS, J.S. Statistical ecology - a priner on methods and computing. New York: John Wiley \& Sons, 1988. 337p.

McGREG0R, S.E. Insect pollination of cultivated crop plants. Washington: USDA, 1976. 411p. (Agriculture Handbook, 496).

MORETI, A.C. de C.C. Estudo sobre a polinização entomófila do girassol (Helianthus annuus L. ) utilizando diferentes métodos de isolamento de flores. 1989. $126 f$. Tese (Doutorado em Entomologia)- Escola Superior de Agricultura “Luiz de Queiroz”, Universidade de São Paulo.

MORETI, A.C. de C.C. Polinização: o principal produto das abelhas. In: CONGRESSO BAIANO DE APICULTURA E ENCONTRO DE MELIPONICULTURA E FEIRA ESTADUAL, 3., 2005, Vitória da Conquista. Anais... Vitória da Conquista: SEAGRI, 2005. p.28-63.

MORETI, A.C. de C.C. et al. Efeito das visitas de abelhas sobre a polinização de três cultivares de girassol (Helianthus annuus), Piracicaba, SP. Boletim de Indústria Animal, Nova Odessa, v.48, n.2, p.83-91, 1991.

MORETI, A.C. de C.C.; MARCHINI, L.C. Observações sobre as abelhas visitantes da cultura do girassol (Helianthus annuus) em Piracicaba-SP. Zootecnia, Nova Odessa, v.30, p.21-27, 1992.

MORGADO, L.N. et al. Fauna de abelhas (Hymenoptera: Apoidea) nas flores de girassol Helianthus annuus L., em Lavras- 
MG. Ciência e Agrotecnologia. Lavras, v.26, n.6, p.11671177, 2002.

ODUM, E.P. Ecologia. Rio de Janeiro: Guanabara Koogan, 1988. 434p.

PAIVA, G.J. de et al. Behavior of Apis mellifera L. Africanized honeybees in sunflower (Helianthus annuus L.) and evaluation of Apis mellifera L. colony inside covered area of sunflower. Acta Scientiarium, Maringá, v.24, n.4, p.851-855, 2002.

PARKER, F.D. How efficient are bees in pollinating sunflowers? Journal of the Kansas Entomological Society, Kansas, v.54, n.1, p.61-67, 1981

REDE BAIANA DE BIOCOMBUSTÍVEL. Oleaginosas da Bahia. Capturado em 19 mar. 2006. Online. Disponível na Internet http://www.redebaianadebiocombustiveis.ba.gov.br/ index.php?menu=oleaginosa.

ROIG-ALSINA, A.; MICHENER. C.D. Studies of the phylogeny and classification of long-tongued bees (Hymenoptera:
Apoidea). University Kansas Science Bulletin, Kansas, v.55, n.4/5, p.123-173, 1994.

SANTOS, F. et al. Diversidade de abelhas (Hymenoptera: Apoidea) em uma área de transição Cerrado-Amazônia. Acta Amazonica, Manaus, v.34, n.2, p. 319-328, 2004.

SHINOHARA, R. et al. Importância da polinização entomófila na cultura do girassol. Zootecnia, Nova Odessa, v.25, n.3, p.275-287, 1987.

SILVA, M.N. da. A cultura do girassol. Jaboticabal: FUNEP, 1990. 67p.

SILVEIRA NETO, S.O. et al. Manual de ecologia dos insetos. Piracicaba: Ceres, 1976. 419p.

SILVEIRA, F.A. et al. Abelhas brasileiras: sistemática e identificação. Belo Horizonte: Composição e Arte, 2002. 253p. 